\title{
Differential attentional guidance by unattended faces expressing positive and negative emotion
}

\author{
JOHN D. EASTWOOD, DANIEL SMILEK, and PHILIP M. MERIKLE \\ University of Waterloo, Waterloo, Ontario, Canada
}

\begin{abstract}
Four experiments were conducted to evaluate whether focal attention can be guided by an analysis of the emotional expression in a face. Participants searched displays of 7, 11, 15, and 19 schematic faces for the location of a unique face expressing either a positive or a negative emotion located among distractor faces expressing a neutral emotion. The slopes of the search functions for locating the negative face were shallower than the slopes of the search functions for locating the positive face (Experiments $1 \mathrm{~A}$ and $2 \mathrm{~A}$ ). When the faces were inverted to reduce holistic face perception, the slopes of the search functions for locating positive and negative faces were not different (Experiments $1 \mathrm{~B}$ and $2 B)$. The results suggest that the emotional expression in a face can be perceived outside the focus of attention and can guide focal attention to the location of the face.
\end{abstract}

A considerable amount of research has established that the visual system is highly efficient at perceiving both faces and facial expressions (e.g., Bruce, Desimone, \& Gross, 1981; Desimone, 1991; Farah, Wilson, Drain, \& Tanaka, 1998; Gorea \& Julesz, 1990; Hasselmo, Rolls, \& Baylis, 1989; Hochberg \& Galper, 1967; Homa, Haver, \& Schwartz, 1976; Purcell \& Stewart, 1988; Schwartz, Izard, \& Ansul, 1985; Tanaka \& Farah, 1993). In fact, it has been suggested that the perception of facial expressions occurs automatically (e.g., Hansen \& Hansen, 1994; Stenberg, Wilking, \& Dahl, 1998) and without conscious awareness (e.g., Morris, Öhman, \& Dolan, 1998). This quick and effortless face perception is thought to be adaptive, given the profound social significance of facial expressions.

One question regarding face perception that remains unanswered is whether the emotion expressed in a face can be perceived outside the focus of attention and can guide focal attention to the location of the face. Recent research indicates that relatively complex types of visual information, such as perceived surfaces (He \& Nakayama, 1992), item structure (Wolfe, 1996), orientations of objects (Enns \& Rensink, 1991), familiarity of objects (Wang, Cavanagh, \& Green, 1994), changes to objects (Smilek, Eastwood, \& Merikle, 2000), and the global representation of a face (Suzuki \& Cavanagh, 1995), can play a functional role in guiding focal attention. It is unclear, however, whether or not emotional expressions are also perceived outside the focus of attention and play a functional role in guiding attention. Such guidance of focal attention would be evident if it was demonstrated

This research was supported by a grant from the Natural Sciences and Engineering Research Council of Canada to P.M.M. We thank Magdalena Stopinski for her assistance in data collection. Correspondence concerning this article should be addressed to J. D. Eastwood, Department of Psychology, University of Waterloo, Waterloo, ON, N2L 3G1, Canada (e-mail: jdeastwo@watarts.uwaterloo.ca). that some facial expressions lead to more attentional guidance than do other facial expressions. Given that the rapid recognition of a potential threat or danger would confer an obvious social and biological advantage, it is conceivable that faces expressing negative emotions might be particularly effective at attracting the focus of attention (e.g., Pratto \& John, 1991).

Visual search methodology is especially well suited for examining whether or not facial expression can be perceived outside the focus of attention and can guide focal attention. If a face expressing either a positive or a negative emotion is embedded among different numbers of distractors and the time taken to locate the target face is measured, the relative contribution of unattended positive and negative facial expressions in guiding focal attention can be assessed by comparing the slopes of the search functions (Smilek et al., 2000). Specifically, as long as the distractor context is held constant and the observers do not know which target to expect on any given trial, the more shallow the slope of the search function, the more effective is unattended information in guiding focal attention (Wolfe, 1994; Wolfe, Cave, \& Franzel, 1989). Therefore, any difference between the slopes of the search functions for locating positive and negative faces would indicate that the emotional expression associated with the shallower slope was the more effective expression for guiding focal attention.

Although the logic of using visual search to assess the effectiveness of negative and positive faces in guiding focal attention is straightforward, to date, applications of visual search methodology have not led to clear conclusions. A number of issues have clouded interpretation of the findings. One issue is that attempts to vary the emotion expressed by the target face have been confounded in some studies with the emotion expressed by the distractor faces. For example, Hansen and Hansen (1988) found that the slope of the search function for locating a target face expressing anger presented among distractor 
faces expressing happiness was shallower than the slope of the search function for locating a target face expressing happiness presented among distractor faces expressing anger. Although these findings suggest that focal attention is more readily guided by an angry face than by a happy face, there is an equally plausible alternative interpretation. Perhaps the reason it took longer to detect the target face expressing happiness than to detect the target face expressing anger is that it takes longer to search through angry distractor faces than it takes to search through happy distractor faces (Hampton, Purcell, Bersine, Hansen, \& Hansen, 1989).

Another issue that has clouded interpretation of the results of studies comparing the effectiveness of different emotional expressions in guiding focal attention is that it has proven difficult to determine whether the observed differences in the speed with which faces expressing different emotions are detected reflects a difference in the emotions expressed by the faces or a difference in the component parts or features that distinguish the faces. By definition, faces expressing different emotions, such as anger and happiness, consist of different composites of features. Given these differences, any evidence showing differential guidance of focal attention by unattended faces expressing different emotions can often be accounted for in terms of the different features, rather than in terms of the different emotions expressed by the faces (e.g., Byrne \& Eysenck, 1995; Nothdurft, 1993; Purcell, Stewart, \& Skov, 1996; White, 1995).

A third issue that has clouded interpretation of previous studies revolves around the question of what constitutes satisfactory evidence that unattended information guides focal attention. In a number of studies, the underlying assumption has been that the only satisfactory evidence that a face guides focal attention is a pattern of findings showing that the speed with which a face is detected is relatively unaffected by the number of distractor faces (e.g., Hampton et al., 1989; Nothdurft, 1993; Purcell et al., 1996; White, 1995). In other words, the slope of the search function across increasing numbers of distractors should be relatively flat. A flat search function showing that a target face pops out when it is embedded in displays of distractor faces certainly provides strong evidence for the role of unattended information in guiding attention. However, a flat search function is not the only evidence that can be used to show the importance of unattended information in guiding focal attention. Another way in which to establish the role of unattended information in guiding attention is to compare the slopes of the search functions for locating different targets. In this way, it is possible to assess whether the different targets lead to relatively more or less guidance of attention (Smilek et al., 2000). Therefore, by comparing the slopes of the search functions for locating faces expressing positive and negative emotions, it is possible to determine whether the positive or the negative emotional expression is the more effective expression for guiding focal attention. ${ }^{1}$
In the present experiments, we compared the slopes of the search functions for locating schematic faces expressing positive and negative emotions in order to assess the relative effectiveness of positive and negative emotional expressions in guiding the focus of attention (see Figure 1 for examples of the stimulus displays). Both the positive and the negative faces were embedded in displays of faces with a neutral emotional expression, to ensure that any differences in the slopes of the search functions were not due to differences in the distractor context (see Hampton et al., 1989). In order to have a sensitive measure of the presence of attentional guidance by faces expressing emotions, the number of distractor faces with a neutral expression was varied widely, with the resulting set sizes ranging from 7 to 19 faces. The participants were required to indicate the spatial location of the target in each display, to ensure that their attention had in fact been directed to the location of the target (see Smilek et al., 2000). Finally, schematic faces were used to minimize featural differences that might overwhelm any potential guidance of focal attention that was based on affective differences between the target faces (see Nothdurft, 1993; Purcell et al., 1996; White, 1995).

\section{EXPERIMENT 1A}

\section{Method}

Participants. Eleven University of Waterloo undergraduate students participated in the experiment in exchange for course credit. Each student had normal or corrected-to-normal vision.

Stimulus displays. Examples of the stimulus displays are shown in Figure 1 . The displays were based on an imaginary $6 \times 6$ matrix that defined 36 possible locations for the targets and the distractors. Each display contained one target, a schematic face expressing either a positive emotion (positive face) or a negative emotion (negative face), and $6,10,14$, or 18 identical distractor faces with a neutral emotional expression (neutral face). On each trial, the target (positive vs. negative face) and the set size (7, 11, 15, or 19) were selected randomly, with the constraint that each condition was tested 30 times across the 240 experimental trials. The locations for the target and the distractors on each trial were also selected randomly.

The displays were presented on an Iiyma Vision Master Pro 17 monitor, which was controlled by a 200-Mhz Pentium processor using Micro Experimental Laboratory software (Schneider, 1990). The imaginary $6 \times 6$ matrix was $106 \mathrm{~mm}$ square and subtended a visual angle of approximately $10.1^{\circ}$ in both the vertical and the horizontal directions at the prescribed viewing distance of $60 \mathrm{~cm}$. Each schematic face was light gray on a dark background, measured $13 \mathrm{~mm}$ in diameter, and subtended a visual angle of approximately $1.2^{\circ}$.

Procedure. The participants were instructed to find the unique image in each display as quickly as possible while maintaining high accuracy. Importantly, the participants were not told which target they would be searching for on any given trial. At the beginning of each trial, a cue was presented on the monitor screen, prompting the participant to press the " $b$ " key to present the display. The displays remained on the screen until the participants pressed the "b" key again to indicate that they had located the unique face. Once the " $b$ " key was pressed, the locations of the faces were covered with gray squares, and column and row numbers (i.e., 1 to 6 ) were presented above and to the left of the display. The participants were instructed to enter the column and row numbers that corresponded to the location of 

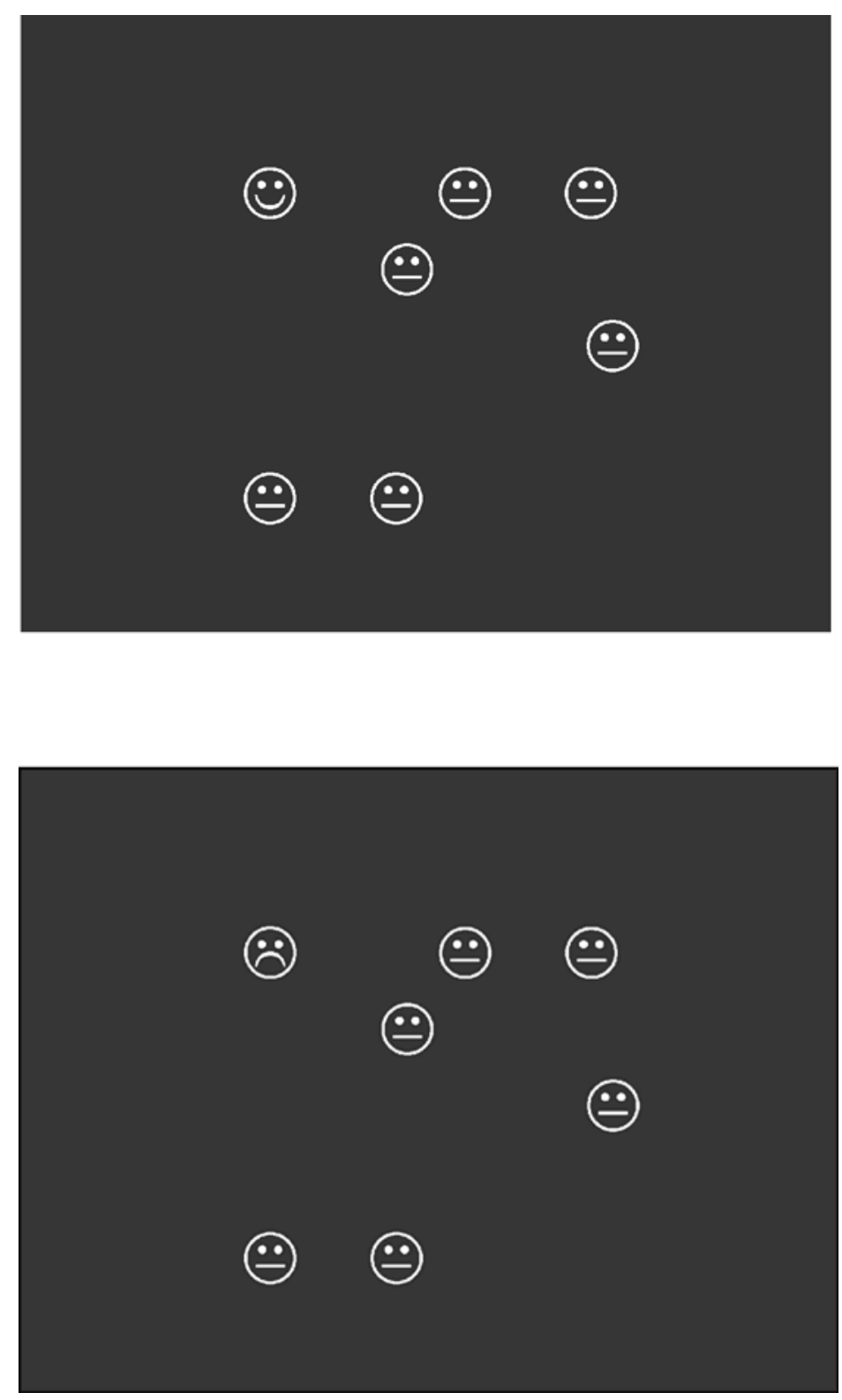

Figure 1. Examples of the stimulus displays used in Experiment 1A.

the unique face. After a 500-msec interval, feedback regarding accuracy was given, and the cue for the next trial was presented.

\section{Results and Discussion}

Reaction time. Before the correct reaction times (RTs) were analyzed, the outliers in each cell were removed, using a recursive procedure (see Van Selst \& Jolicœur, 1994). The highest and lowest RTs were removed, one at a time, and the mean and the standard deviation $(S D)$ of the resulting distribution were calculated. If the extreme $\mathrm{RT}$ was more than four $S D$ s from the mean, it was considered an outlier and was removed. This procedure was repeated until no outliers remained. A total of $2.02 \%$ of the trials were removed in this manner. The remaining RT data were then evaluated by a $2 \times 4$ analysis of variance (ANOVA) to assess target type (positive vs. negative face) and set size (7, 11, 15, and 19).

Figure 2 shows the mean RTs to locate the positive and negative faces for each set size. As is suggested by the figure, negative faces were located faster than positive faces $\left[F(1,10)=25.82, M S_{\mathrm{e}}=20,652, p<.001\right]$, and search became more difficult with increased set size $\left[F(3,30)=25.92, M S_{\mathrm{e}}=6,380.57, p<.001\right]$. To establish whether the positive and the negative faces led to differential guidance of focal attention, the linear components of the search functions were compared. This 


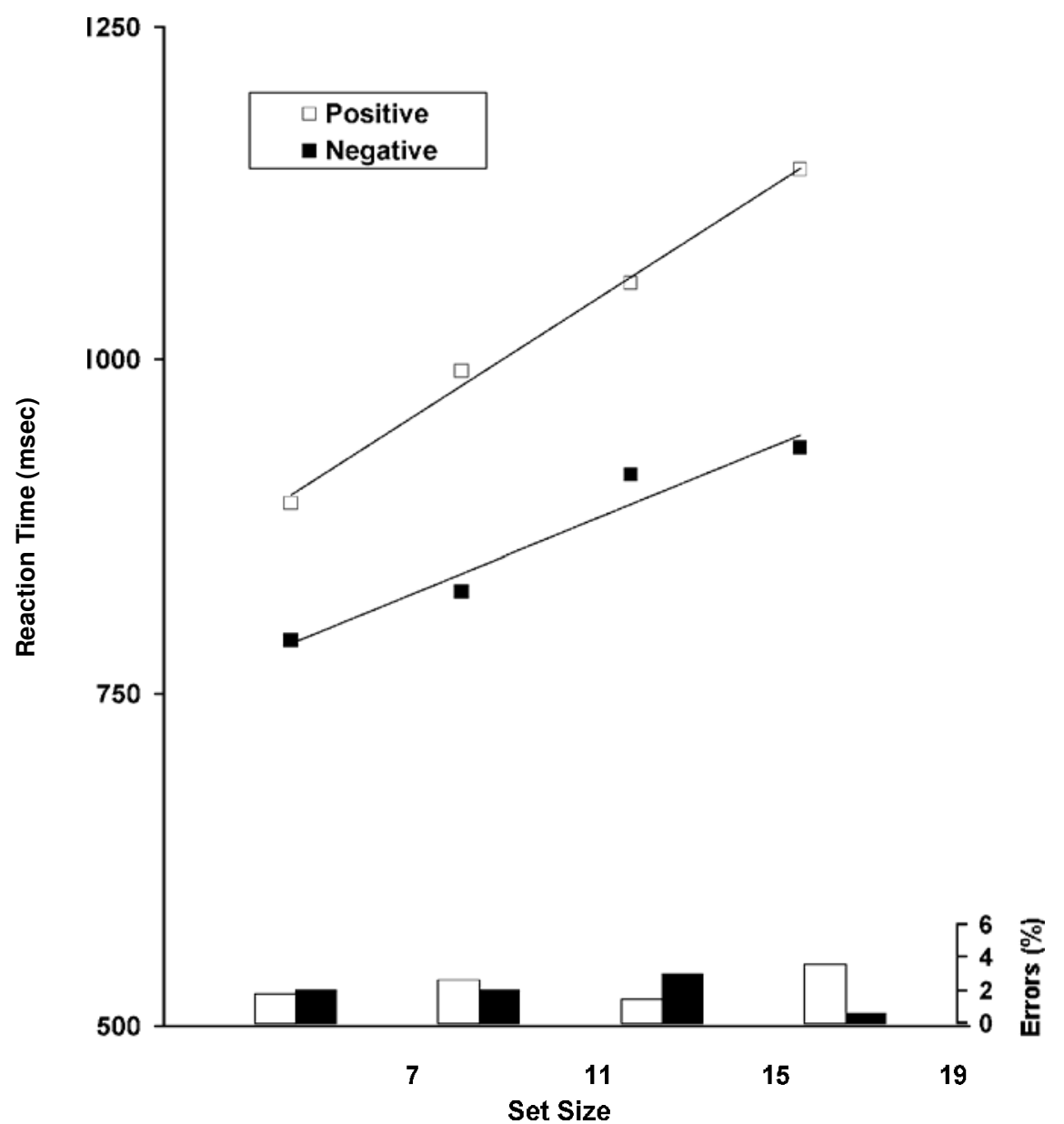

Figure 2. Mean reaction times and errors for detecting the positive and negative faces in Experiment 1A.

comparison revealed that the slope of the search function for locating the negative face $(S=13.0 \mathrm{msec} / \mathrm{item})$ was shallower than the slope of the search function for locating the positive face $[S=20.5 \mathrm{msec} /$ item; $F(1,10)=$ $\left.5.32, M S_{\mathrm{e}}=4,552.45, p=.04\right]$. Given the logic of visual search, the shallower slope of the search function for locating the negative face indicates that the negative face guided focal attention better than did the positive face. Thus, the results suggest that positive and negative faces are differentially effective in guiding focal attention when people are asked to search for the location of a unique face among neutral face distractors.

Error data. The mean error rates for each condition are shown at the bottom of Figure 2. All error rates were less than 4\%, and an ANOVA revealed neither significant main effects for target type (positive vs. negative face) or set size $(7,11,15$, and 19) nor a significant interaction between the linear components of the search functions (all $F \mathrm{~s}<2.8, p>.10$ ). Thus, interpretation of the RT data does not appear to have been compromised by speed/ accuracy tradeoffs.

\section{EXPERIMENT 1B}

The purpose of Experiment 1B was to determine whether the differential guidance of focal attention that was observed in Experiment 1A occurred because the negative and the positive faces had different affective valences or because the faces had different component parts or features (e.g., downward curved arc or upward curved arc). Given the confound between affective valence and features, the results of Experiment 1A can be interpreted as showing either that the negative face guided focal attention better than did the positive face or that the downward curved arc guided focal attention better than did the upward curved arc. To rule out the possibility that the obtained slope differences in Experiment 1A were mediated by featural differences, the target faces were inverted in Experiment 1B. If the differential guidance of focal attention observed in Experiment 1A was due to featural differences between the negative and the positive faces, search slope differences should be maintained in Experiment 1B, because inverting the faces retains the 
featural differences between the negative and the positive faces. However, if faces are represented holistically, with relatively little part decomposition, as has been claimed by a number of investigators (e.g., Farah, Tanaka, \& Drain, 1995; Farah et al., 1998; Moscovitch, Winocur, \& Behrmann, 1997), inverting the target faces should disrupt the perception of emotional expression (see Köhler, 1940; Yin, 1969). Consequently, if the differential guidance of focal attention that was observed in Experiment 1A was due to perception of emotional expression, no differences in the slopes of the search functions for the negative and the positive faces should be found in Experiment 1B.

\section{Method}

Participants. Eleven University of Waterloo undergraduate students participated in the experiment in exchange for course credit. Each student had normal or corrected-to-normal vision.

Stimulus displays. With one important exception, the stimulus displays were the same as the displays used in Experiment $1 \mathrm{~A}$. Rather than presenting the targets and distractors in an upright orientation, the targets and distractors were rotated $180^{\circ}$. The other aspects of the displays and all the details regarding display presentation were the same as those in Experiment 1A.
Procedure. The procedure was the same as that followed in Experiment $1 \mathrm{~A}$.

\section{Results and Discussion}

Reaction time. As in Experiment 1A, the outliers in each cell were removed, using a recursive procedure. A total of $1.40 \%$ of the trials were removed in this manner. The remaining data were then evaluated by an ANOVA to assess target (positive vs. negative face) and set size $(7,11,15$, and 19).

Figure 3 shows the mean RTs to locate the inverted positive and negative faces for each set size. As in Experiment $1 \mathrm{~A}$, negative faces were located faster than positive faces $\left[F(1,10)=9.44, M S_{\mathrm{e}}=3,322.95, p<.02\right]$, and search became more difficult with increased set size $\left[F(3,30)=21.16, M S_{\mathrm{e}}=7,445.18, p<.001\right]$. However, in contrast to the results of Experiment 1A, there was no interaction between the linear components of the search functions for locating the positive $(S=16.8 \mathrm{msec} / \mathrm{item})$ and the negative $(S=15.5 \mathrm{msec} /$ item $)$ faces $[F(1,10)=$ $\left.0.33, M S_{\mathrm{e}}=2,628.37, p=.581\right]$. Thus, the results provide no evidence that the search function for inverted negative faces was shallower than the search function for inverted

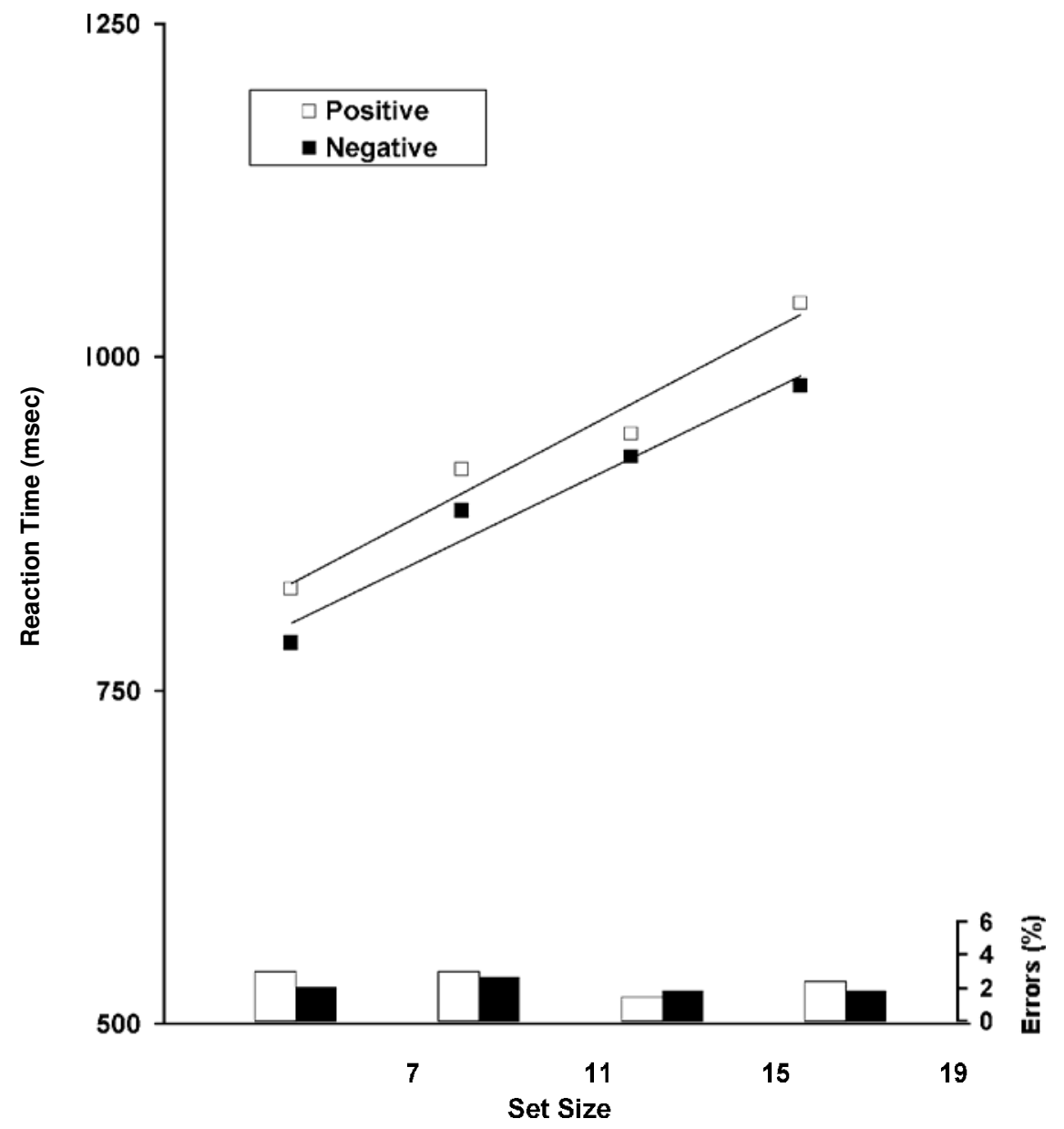

Figure 3. Mean reaction times and errors for detecting the inverted positive and negative faces in Experiment 1B. 
positive faces. The absence of a significant difference between the slopes of the search functions for locating inverted positive and negative faces suggests that the differential guidance of focal attention observed in Experiment $1 \mathrm{~A}$ for upright positive and negative faces was the result of the difference in affective valence between the positive and the negative faces, and not the result of featural differences between the faces.

One issue that needs to be addressed concerns the similarity of the slopes of the search functions in Experiments $1 \mathrm{~A}$ and 1B. For example, the slope of the search function for locating the upright negative face in Experiment 1A (13.0 msec/item) was similar to the slope of the search function for locating an inverted negative face in Experiment 1B (15.5 msec/item). If an upright negative face attracts attention more effectively than does an upright positive face, one might expect that an upright negative face would also attract attention more effectively than would an inverted negative face. In other words, the slope of the search function for locating an upright negative face might be shallower than the slope of the search function for locating an inverted negative face. The present findings cannot be used to evaluate this expectation, because it is not possible to make meaningful comparisons across Experiments 1A and 1B, owing to the fact that, in addition to the difference in the targets between experiments, there were also differences in the participants' expectations between experiments. Therefore, although the results of the present experiments can be used to assess the differential guidance of attention by upright positive and negative faces (Experiment 1A) and inverted positive and negative faces (Experiment 1B), the results cannot be used to compare the absolute sizes of the slope functions across experiments.

Error data. The mean error rates for each condition are shown at the bottom of Figure 3. The error rates were less than $4 \%$ in all the conditions, and an ANOVA revealed neither significant main effects for target (positive vs. negative face) or set size $(7,11,15$, and 19) nor a significant interaction between the linear components of the search functions (all $F_{\mathrm{S}}<0.90, p>.46$ ). Thus, interpretation of the RT data does not appear to have been compromised by speed/accuracy tradeoffs.

\section{EXPERIMENTS 2A AND 2B}

The purpose of Experiments $2 \mathrm{~A}$ and $2 \mathrm{~B}$ was to replicate the results of Experiments $1 \mathrm{~A}$ and $1 \mathrm{~B}$, using a distractor context that remained constant, independent of whether the negative and positive target faces were upright or inverted. One limitation to drawing conclusions on the basis of a comparison between the results of Experiments $1 \mathrm{~A}$ and $1 \mathrm{~B}$ is that the experiments differed not only in terms of whether the target faces were upright or inverted, but also in terms of whether the neutral distractor faces were upright or inverted. Given that visual search is influenced by both the targets and the distractors, conclusions regarding the relative effectiveness of upright and inverted emotional faces in attracting focal attention would be stronger if the upright and the inverted target faces were presented in the same distractor context. For this reason, in Experiments $2 \mathrm{~A}$ and 2B, half of the neutral distractor faces for each set size were upright, and the other half of the distractor faces were inverted. As in Experiments $1 \mathrm{~A}$ and $1 \mathrm{~B}$, the critical difference between Experiments $2 \mathrm{~A}$ and $2 \mathrm{~B}$ was that the target faces were upright in Experiment $2 \mathrm{~A}$ and inverted in Experiment $2 \mathrm{~B}$. If the pattern of results across Experiments $2 \mathrm{~A}$ and $2 \mathrm{~B}$ is similar to the pattern of results across Experiments $1 \mathrm{~A}$ and $1 \mathrm{~B}$, this would provide further evidence for the conclusion that upright negative faces guide focal attention more effectively than do upright positive faces.

\section{Method}

Participants. Eleven University of Waterloo undergraduate students participated in each experiment in exchange for course credit. Each student had normal or corrected-to-normal vision.

Stimulus displays. In both experiments, half of the neutral face distractors in each display were upright, and the other half of the distractors in each display were inverted. In Experiment 2A, the targets were upright positive and negative faces, whereas in Experiment $2 \mathrm{~B}$, the targets were inverted positive and negative faces. The other aspects of the displays and all the details regarding presentation of the displays were the same as those in Experiment 1A.

Procedure. The procedure was the same as that followed in Experiments $1 \mathrm{~A}$.

\section{Results and Discussion}

Reaction time. As in the previous experiments, the outliers in each cell were removed, using a recursive procedure. A total of $2.25 \%$ of the trials in Experiment $2 \mathrm{~A}$ and $2.17 \%$ of the trials in Experiment $2 \mathrm{~B}$ were removed in this manner. The RTs for each experiment were then evaluated by an ANOVA to assess target (positive vs. negative face) and set size (7, 11, 15, and 19).

The mean RTs to locate the positive and negative faces in Experiment 2A and 2B are shown in Figures 4 and 5, respectively. As is shown in these figures, negative faces were easier to detect than positive faces in both Experiment $2 \mathrm{~A}\left[F(1,10)=15.76, M S_{\mathrm{e}}=37,184, p<.004\right]$ and Experiment $2 \mathrm{~B}\left[F(1,10)=18.15, M S_{\mathrm{e}}=12,946, p<\right.$ $.05]$. Figures 4 and 5 also show that search became more difficult with increased set size in both Experiment $2 \mathrm{~A}$ $\left[F(3,30)=20.38, M S_{\mathrm{e}}=16,164, p<.001\right]$ and Experiment 2B $\left[F(3,30)=26.08, M S_{\mathrm{e}}=7,067, p<.01\right]$.

The critical comparisons between Experiments $2 \mathrm{~A}$ and 2B involved the slopes of the search functions for the negative and positive faces. In Experiment $2 \mathrm{~A}$ (Figure 4), the slope of the search function for locating the upright negative face $(S=15.7 \mathrm{msec} /$ item) was shallower than the slope of the search function for locating the upright positive face $\left[S=30.4 \mathrm{msec} /\right.$ item; $F(1,10)=7.78, M S_{\mathrm{e}}=$ $12,369, p=.02]$. This interaction is similar to the interaction found in Experiment $1 \mathrm{~A}$ and indicates that the negative face guided focal attention more effectively than did the positive face. In contrast, the slopes of the search functions in Experiment 2B (Figure 5) for locating the inverted negative $(S=15.1 \mathrm{msec} /$ item $)$ and pos- 


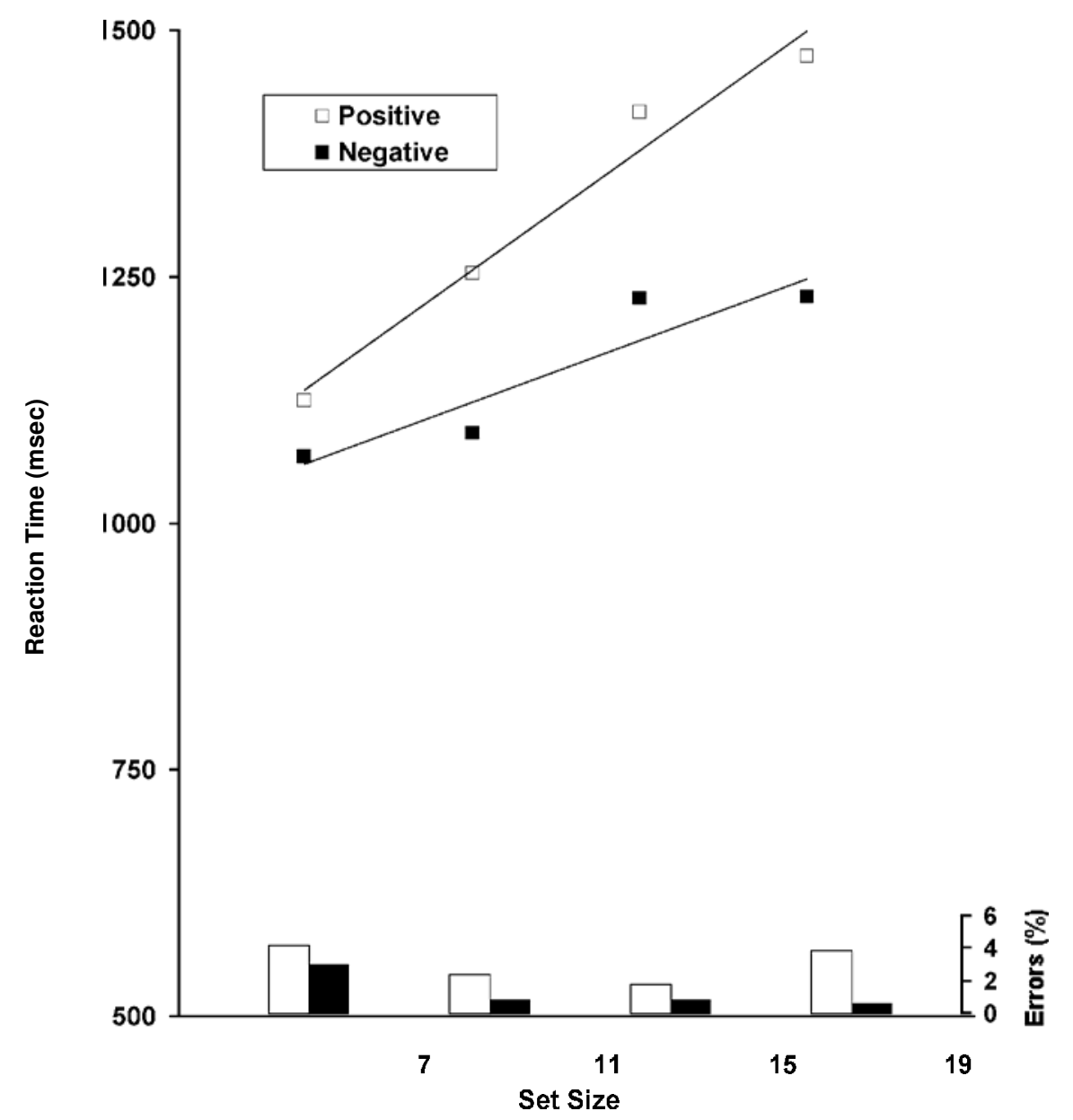

Figure 4. Mean reaction times and errors for detecting the upright positive and negative faces in Experiment 2A.

itive ( $S=20.3 \mathrm{msec} /$ item $)$ faces were similar and did not differ significantly $\left[F(1,10)=2.44, M S_{\mathrm{e}}=4,978.35, p>\right.$ .14]. This absence of any difference between the slopes of the search functions for locating inverted positive and negative faces is consistent with the results found in Experiment $1 \mathrm{~B}$ and indicates that the inverted positive and negative faces did not lead to differential guidance of focal attention. Overall, the results of Experiments 2A and $2 \mathrm{~B}$ replicate the findings from Experiments $1 \mathrm{~A}$ and $1 \mathrm{~B}$ under conditions in which the distractor context was held constant and only the orientation of the target faces was varied.

To further evaluate the present findings, the RT data from Experiments 1A, 1B, 2A, and 2B were combined and subjected to an ANOVA that assessed target orientation (upright vs. inverted), target type (positive vs. negative), and set size $(7,11,15$, and 19). The analysis revealed a significant three-way interaction among the linear components of the search functions $[F(1,42)=$ $\left.4.29, M S_{\mathrm{e}}=6,202.71, p<.05\right]$. This interaction indicates that the difference between the slopes of the search functions for locating positive and negative faces was significantly larger when the target faces were upright (Experiments $1 \mathrm{~A}$ and $2 \mathrm{~A}$ ) than when the target faces were inverted (Experiments $1 \mathrm{~B}$ and $2 \mathrm{~B}$ ). Thus, this analysis of the combined data provides further support for the conclusion that faces expressing negative emotion guide attention more effectively than do faces expressing positive emotion and that this difference between positive and negative faces is not the result of feature differences between the faces.

Error data. The error rates for each condition of Experiments $2 \mathrm{~A}$ and $2 \mathrm{~B}$ are shown at the bottom of Figures 4 and 5. All error rates in both experiments were less than $4 \%$. For Experiment 2A, an ANOVA revealed significant main effects for both target type [positive vs. negative face; $\left.F(1,10)=8.85, M S_{\mathrm{e}}=0.00076, p=.01\right]$ and set size $\left[7,11,15\right.$, and $19 ; F(3,30)=3.16, M S_{\mathrm{e}}=0.00071$, $p=.04]$. However, the analysis also revealed that the interaction between target type and set size was not significant $\left(F=1.00, M S_{\mathrm{e}}=0.00089\right)$. For Experiment $2 \mathrm{~B}$, an ANOVA revealed neither significant main effects for target type or set size nor a significant interaction between target type and set size (all $\left.F_{\mathrm{S}}<1.30\right)$. The absence of any 


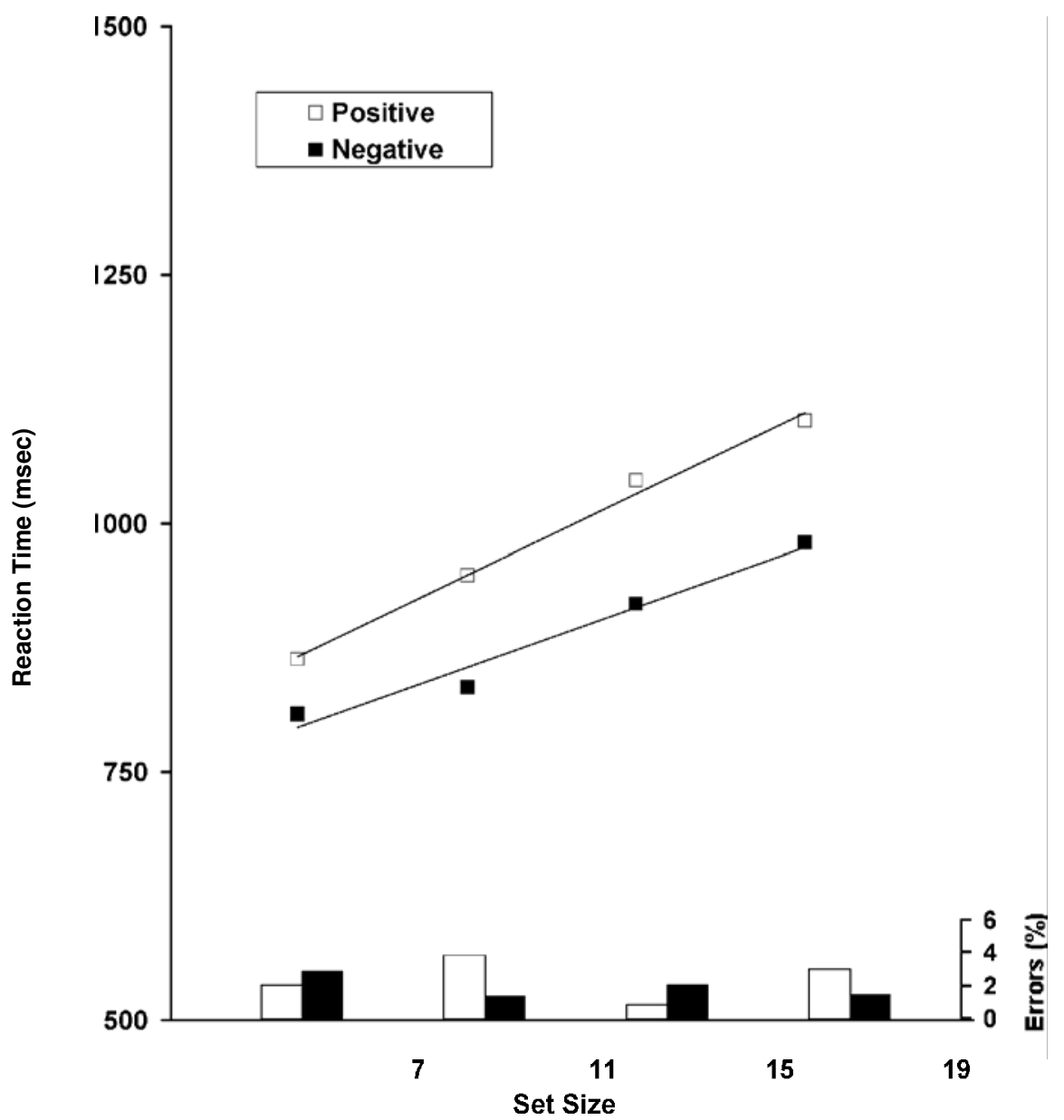

Figure 5. Mean reaction times and errors for detecting the inverted positive and negative faces in Experiment 2B.

interactions between target type and set size suggests that interpretation of the RT data was not compromised by speed/accuracy tradeoffs.

\section{GENERAL DISCUSSION}

In the present experiments, we examined whether unattended schematic faces expressing positive and negative emotion differ in the relative effectiveness with which they guide focal attention. The results of Experiments $1 \mathrm{~A}$ and $2 \mathrm{~A}$ show that the slopes of the search functions for locating negative faces embedded in a context of neutral faces were shallower than the slopes of the search functions for locating positive faces embedded in a context of neutral faces. Given the assumption that shallower slopes reflect relatively greater guidance of the focus of attention, these results indicate that faces expressing negative emotion guide focal attention more effectively than do faces expressing positive emotion, when the faces are presented in the context of neutral faces. The results thus support the conclusion that the emotion expressed in a face can be perceived outside the focus of attention and that the perceived emotion can subsequently guide the focus of attention to the location of the face.

The results of Experiments $1 \mathrm{~B}$ and $2 \mathrm{~B}$ show that the differential attentional guidance by upright positive and negative faces found in Experiments 1A and 2A was not based on differences in the component parts of the faces. If the differential guidance of focal attention was simply due to part differences, rather than to holistic face perception, the slopes of the search functions for locating inverted positive and negative faces should have been different. However, the results of Experiments $1 \mathrm{~B}$ and $2 \mathrm{~B}$ revealed that the slopes of the search functions for locating the inverted positive and negative faces were similar. Given the similar slopes of the search functions for locating inverted positive and negative faces, it is unlikely that the differential guidance of focal attention by positive and negative faces found in Experiments $1 \mathrm{~A}$ and $2 \mathrm{~A}$ can be accounted for in terms of differences between the component parts of the faces.

Although the schematic face images used in the present study were impoverished, as compared with photographs of real human faces, they appeared to function as 
potent affective facial stimuli. This is consistent with a number of findings in the literature. For example, schematic faces appear to communicate emotional meaning effectively (Aronoff, Barclay, \& Stevenson, 1988; Cuceloglu, 1970; McKelvie, 1973). Schematic faces also show disruptions in perception when inverted (Farah et al., 1998; Moscovitch et al., 1997; Yin, 1969) similar to those found with photographed faces. Furthermore, it has recently been shown that schematic faces elicit eventrelated potentials that are similar to those elicited by photographs of faces (Sagiv \& Bentin, in press). Therefore, given that schematic faces contain fewer feature confounds than do photographed faces and yet appear to function as effective affective face stimuli, it may actually be preferable to use schematic faces in studies in which the perception of facial expression is investigated.

The present findings are consistent with a growing body of literature suggesting that potentially threatening or dangerous stimuli are perceived very efficiently and, in general, demand attention (see Taylor, 1991, for a review). LeDoux (1996), for example, argues for a direct neural pathway from the sensory thalamus to the amygdala, which is able to support rapid and defensive responses to potentially dangerous stimuli, even before conscious identification and evaluation of the stimuli. Developmental research has also demonstrated that infants are efficient at noticing and recognizing negative facial expressions (e.g., Sackett, 1966; Schwartz et al., 1985). Furthermore, on the basis of investigations of monkeys reared in isolation, Sackett claims that pictures of animals of the same species displaying threat "appear to have prepotent general activating properties ... and release a developmentally determined, inborn fear response" (p. 1468). In light of the important role that faces can play in communicating potential negative outcomes, it is perhaps not surprising that negative facial expressions appear to be particularly effective at drawing attention to themselves.

Negative stimuli, such as faces expressing negative emotion, also appear to be a more potent source of involuntary interference to ongoing cognitive tasks than are positive stimuli (Pratto \& John, 1991; White, 1996). For example, White (1996) showed that a nonattended, irrelevant angry face interfered with the completion of an ongoing task, whereas a happy face did not disrupt performance. Pratto and John used linguistic stimuli to demonstrate what they termed "automatic vigilance," which occurs whenever "attentional resources are automatically directed away from an attended task to undesirable stimuli" (p. 380).

There are, however, findings reported by Mack and Rock (1998) that are an exception to the general pattern of findings reported in the literature. On each trial of their experiments, participants were required to judge which arm of a parafoveally presented cross was longer. Unknown to the participants, on one trial of the experiment, a schematic face was presented at fixation at the same time as the cross. The results showed that when the face expressed a positive emotion, very few of the participants failed to detect it; however, when the face expressed a negative emotion, significantly more participants failed to detect its presence. These results seem to be inconsistent with the idea that faces expressing negative emotion guide focal attention to themselves more effectively than do faces expressing positive emotion when they are presented in the context of neutral faces.

At this time, it is not clear how to reconcile Mack and Rock's (1998) findings with either the present findings or other findings in the literature regarding the impact of emotional stimuli on attentional processes. However, there are at least two factors that may account for the discrepancy between Mack and Rock's results and the findings reported more generally in the literature. First, Mack and Rock acknowledge that for the schematic faces used in their studies, the happy face may have been more familiar than the sad face (p. 143). If so, it is very possible that the more familiar happy face may have attracted attention and been detected more readily than the less familiar sad face. The second factor that may account for Mack and Rock's findings is a difference in the salience of the features associated with the happy and the sad faces. Mack and Rock report that a modified stimulus consisting solely of the outer contour and the "mouth" of the happy face captured attention as well as the regular happy face (pp. 144-145) and that the frequency with which a scrambled happy face captured attention did not differ significantly from the frequency with which a regular happy face captured attention (pp. 140-142). These findings suggest that the salience of the curved line used to represent the mouth in a schematic happy face may have been the factor that made the happy face so effective for capturing attention. Although neither familiarity nor salience may eventually account for the discrepancy between Mack and Rock's results and the more typical results found in the literature, these factors are likely candidates for explaining why Mack and Rock's findings regarding the impact of happy and sad faces on attentional processes are inconsistent with the findings in so many other studies investigating the impact of emotional stimuli on attentional processes.

Our conclusion that attention can be guided by unattended faces expressing emotion is consistent with other findings showing that visual search is influenced by the global representations of faces that are formed by composites of parts. In a recent study, Suzuki and Cavanagh (1995) compared visual search for a unique feature (e.g., down arc) when it was located in 6,12 , or 18 triplets of features (e.g., up arcs) arranged to form either faces or meaningless patterns. They found that visual search for the unique feature was less efficient, as was indicated by the slopes of the search functions, when the features were arranged to form faces. On the basis of these results, Suzuki and Cavanagh concluded that a global representation of a face can have priority during visual search and that the processing of the global representation can even preempt access to such features as the curvature of an arc. The present findings extend these conclusions by suggesting not only that faces are perceived outside of the 
focus of attention, but also that emotional expressions are perceived outside of the focus of attention and play a functional role in guiding focal attention.

\section{REFERENCES}

Aronoff, J., Barclay, A. M., \& Stevenson, L. A. (1988). The recognition of threatening facial stimuli. Journal of Personality \& Social Psychology, 54, 647-655.

Bruce, C., Desimone, R. \& Gross, C. G. (1981). Visual properties of neurons in a polysensory area in superior temporal sulcus of the macaque. Journal of Neurophysiology, 46, 369-384.

Byrne, A., \& Eysenck, M. W. (1995) Trait anxiety, anxious mood, and threat detection. Cognition \& Emotion, 9, 549-562.

Cuceloglu, D. M. (1970). Perception of facial expressions in three different cultures. Ergonomics, 13, 93-100.

Desimone, R. (1991). Face selective cells in temporal cortex of monkeys. Journal of Cognitive Neuroscience, 3, 1-8.

Enns, J. T., \& Rensink, R. A. (1991). Preattentive recovery of threedimensional orientation from line drawings. Psychological Review, 98, 335-351.

Farah, M. J., Tanaka, J. W., \& Drain, H. M. (1995). What causes the face inversion effect? Journal of Experimental Psychology: Human Perception \& Performance, 21, 628-634.

Farah, M. J., Wilson, K. D., Drain, M., \& Tanaka, J. N. (1998). What is "special" about face perception? Psychological Review, 105, 482-498.

Gorea, A., \& Julesz, B. (1990). Context superiority in a detection task with line-element stimuli: A low-level effect. Perception, 19, 5-16.

Hampton, C., Purcell, D. G., Bersine, L., Hansen, C. H., \& Hansen, R. D. (1989). Probing "pop-out": Another look at the face-in-thecrowd effect. Bulletin of the Psychonomic Society, 27, 563-566.

HANSEN, C. H., \& HANSEN, R. D. (1988). Finding the face in the crowd: An anger superiority effect. Journal of Personality \& Social Psychology, 54, 917-924.

HAnsen, C. H. \& HANSEN, R. D. (1994). Automatic emotion: Attention and facial efference. In P. M. Niedenthal \& S. Kitayama (Eds.), The heart's eye: Emotional influences in perception and attention (pp. 217-243). San Diego: Academic Press.

Hasselmo, M. E., Rolls, E. T., \& Baylis, G. C. (1989). The role of expression and identity in the face-selective responses of neurons in the temporal visual cortex of the monkey. Behavioural Brain Research, 32, 203-218.

He, Z J., \& NaKayama, K. (1992). Surface versus features in visual search. Nature, 359, 231-233.

Hochberg, J., \& Galper, R. E. (1967). Recognition of faces: I. An exploratory study. Psychonomic Science, 9, 619-620.

Homa, D., Haver, B., \& Schwartz, T. (1976). Perceptibility of schematic face stimuli: Evidence for a perceptual gestalt. Memory \& Cognition, 4, 176-185.

KöHLER, W. (1940). Dynamics in psychology. New York: Liveright.

LeDoux, J. (1996). The emotional brain. New York: Touchstone.

MACK, A., \& Rock, I. (1998). Inattentionalblindness. Cambridge, MA: MIT Press.

McKelvie, S. J. (1973). The meaningfulness and meaning of schematic faces. Perception \& Psychophysics, 14, 343-348.

Morris, J. S., Öhman, A., \& Dolan, R. J. (1998). Conscious and unconscious emotional learning in the human amygdala. Nature, 393, 467-470.

Moscovitch, M., Winocur, G., \& Behrmann, M. (1997). What is special about face recognition? Nineteen experiments on a person with visual object agnosia and dyslexia but normal face recognition. Journal of Cognitive Neuroscience, 9, 555-604.

Nothdurft, H.-C. (1993). Faces and facial expressions do not pop out. Perception, 22, 1287-1298.

Pratto, F., \& John, O. P. (1991). Automatic vigilance: The attention- grabbing power of negative social information. Journal of Personality \& Social Psychology, 61, 380-391.

Purcell, D. G., \& STEWART, A. L. (1988). The face-detection effect: Configuration enhances detection. Perception \& Psychophysics, 43, 355-366.

Purcell, D. G., Stewart, A. L., \& Skov, R. B. (1996). It takes a confounded face to pop out of a crowd. Perception, 25, 1091-1108.

SACKeTt, G. P. (1966). Monkeys reared in isolation with pictures as visual input: Evidence for an innate releasing mechanism. Science, 154, 1468-1473.

SAgiv, N., \& Bentin, S. (in press). Structural encoding of human and schematic faces: Holistic and part-based processes. Journal of Cognitive Neuroscience.

SCHNEIDER, W. (1990). Mel users guide: Computer techniques for realtime experimentation. Pittsburgh: Psychology Software Tools.

Schwartz, G. M., Izard, C. E., \& Ansul, S. E. (1985). The 5-monthold's ability to discriminate facial expressions of emotion. Infant Behaviour \& Development, 8, 65-77.

Smilek, D., Eastwood, J. D., \& Merikle, P. (2000). Does unattended information facilitate change detection? Journal of Experimental Psychology: Human Perception \& Performance, 26, 480-487.

Stenberg, G., Wilking, S., \& DAhl, M. (1998). Judging words at face value: Interference in a word processing task reveals automatic processing of affective facial expressions. Cognition \& Emotion, 12, 755-782.

Suzuki, S., \& CAVAnagh, P. (1995). Facial organization blocks access to low-level features: An object inferiority effect. Journal of Experimental Psychology: Human Perception \& Performance, 21, 901-913.

TANAKA, J. W., \& FARAH, M. J. (1993). Parts and wholes in face recognition. Quarterly Journal of Experimental Psychology, 46A, 225-245.

TAYLOR, S. E. (1991). Asymmetrical effects of positive and negative events: The mobilization minimization hypothesis. Psychological Bulletin, 110, 67-85.

VAN Selst, M., \& Jolicceur, P. (1994). A solution to the effect of sample size on outlier elimination. Quarterly Journal of Experimental Psychology, 47A, 631-650.

Wang, Q., Cavanagh, P., \& GReen, M. (1994). Familiarity and pop-out in visual search. Perception \& Psychophysics, 56, 495-500.

White, M. (1995). Preattentive analysis of facial expressions of emotion. Cognition \& Emotion, 9, 439-460.

White, M. (1996). Anger recognition is independent of spatial attention. New Zealand Journal of Psychology, 25, 30-35.

Wolfe, J. M. (1994). Guided Search 2.0: A revised model of visual search. Psychonomic Bulletin \& Review, 1, 202-238.

WolfE, J. M. (1996). Extending guided search: Why guided search needs a preattentive "item map." In A. F. Kramer, M. G. H. Coles, \& G. D. Logan (Eds.), Converging operations in the study of visual selective attention (pp. 247-267). Washington, DC: American Psychological Association.

Wolfe, J. M., CAVE, K. R, \& Franzel, S. L. (1989). Guided Search: An alternative to the feature integ ration model for visual search. Journal of Experimental Psychology: Human Perception \& Performance, 15, 419-433.

YIN, R. K. (1969). Looking at upside-down faces. Journal of Experimental Psychology, 81, 141-145.

\section{NOTE}

1. Byrne and Eysenck (1995) used a visual-search-like task to study threat detection in high and low anxious individuals. However, Byrne and Eysenck did not vary set size. For this reason, it is not possible to compute search slopes on the basis of their findings.

(Manuscript received April 5, 2000; revision accepted for publication December 27, 2000.) 